\title{
Revolución Cubana y Educación Superior
}

\author{
Sinesio C. Santos Gutiérrez ${ }^{1}$ \\ Francisco LóPEz Segrera ${ }^{2}$
}

Recebido em: 14/03/07

Avaliado em: 25/05/07

Resumen: Los autores elaboran un panorama de la educación superior cubana de los últimos años, subrayando los desafíos económicos y políticos debidos a las dificultades que el gobierno de Cuba está enfrentando desde 1989 (la desaparición del campo socialista; el recrudecimiento del bloqueo de EE.UU, la crisis económica y social del Sur; la "victoria del neoliberalismo", la necesidad de insertarse en un nuevo tipo de mercado mundial global). El artículo analiza las demandas e indicadores de la educación superior cubana, realzando las transformaciones que ocurren especialmente a partir del año 2001 respecto al aumento de la equidad, políticas de inclusión y consolidación de un nuevo modelo de ciencia y tecnología. Además, los autores presentan una tipología de la educación superior y consideran sus impactos en la sociedad cubana.

Palavras-chave: Cuba; Educação superior; Papel social da Universidade; Ciência e Tecnologia.

The Cuban Revolution and Higher Education

\begin{abstract}
The authors trace a view of Cuban higher education in recent years, emphasizing the economic and political challenges due to the difficulties faced by Cuba since 1989 at national and international levels (disappearance of the socialist field, tightening of the American blockade, the social and economic crisis of the South, the "victory of neoliberalism", the need to insert itself in a new type of global market, etc.). The article analyses the demands and indicators of Cuban higher education, stressing the transformations that have occurred particularly after 2001, in relation to the increase of equity and inclusion policies, as well as the consolidation of a new model of science and technology. Furthermore, the authors present a typology of higher education and consider its impacts on the Cuban society.
\end{abstract}

Key-words: Cuba; Higher Education; Social Role of the University; Science and Technology.

\footnotetext{
${ }^{1}$ Profesor e Investigador del Centro de Estudios para el Perfeccionamiento de la Educación Superior de la Universidad de la Habana. Vicerrector de la Universidad de la Habana (1990-1994). Autor de numerosos artículos sobre la educación superior cubana. Email: ssantos@cepes.uh.cu

${ }^{2}$ Asesor Académico de la Global University Network for Innovation (GUNI). Editor de "La Educación Superior en el Mundo". Miembro del Foro UNESCO de educación superior, investigación y conocimiento. Miembro del Grupo de Trabajo de CLACSO sobre Universidad y Sociedad. Director del IESALC (1999-2001). Investigador Titular Adjunto del Centro Juan Marinello (Cuba) Email: francisco.lopez-segrera@upc.edu
} 


\section{Desafíos del Período 1990-2006}

Desde 1989 el gobierno cubano está enfrentando enormes desafíos: la desaparición del campo socialista; el recrudecimiento del bloqueo de EE.UU.; la emergencia de un mundo unipolar liderado por EE.UU.; la crisis económica y social del Sur; la "victoria del neoliberalismo"; las secuelas del 11 de septiembre y de la actual recesión mundial; y la urgente necesidad de insertarse en un nuevo tipo de mercado mundial global.

Cuba ha resistido y continuado sus diversos programas de desarrollo y construcción de la equidad, entre ellos el de la educación superior, en este difícil entorno. En los últimos años se han llevado cabo importantes reformas económicas y sociales. El gobierno cubano ha anunciado como objetivo prioritario el reducir las desigualdades aparecidas durante el "periodo especial", que se inició en 1990 y se ha hecho un gran énfasis en la lucha contra la corrupción a todos los niveles.

El crecimiento del PIB del 2005 según la CEPAL fue de alrededor del 5\%. El gobierno cubano, según una metodología propia, estimó un crecimiento del 11,8\%. Conforme a dicho método, cuyo objetivo es reflejar la "verdadera realidad de la economía cubana", el PIB nacional debe incluir los Servicios Sociales Gratuitos ofertados a terceros países en áreas como la salud y la educación y prestados por profesionales cubanos desplazados a esos países. La tasa de crecimiento se ha alcanzado pese a las medidas de EEUU restringiendo las remesas, los daños de los huracanes, la sequía y la escasez de energía eléctrica, que han afectado negativamente a la economía cubana en el 2005.

\section{Análisis de la Demanda de Educación Superior}

\subsection{Niveles educativos previos a la educación terciaria}

La educación general es pública y gratuita, con un nivel de cobertura nacional y total, pues el país habilita cada año suficientes capacidades para absorber toda la demanda en todos los niveles educativos desde el nivel preescolar hasta el preuniversitario o el nivel medio profesional.

La tasa de alfabetización del segmento de la población comprendido entre 15 y 24 años es de $99.96 \%$. El $77.58 \%$ del segmento de la población entre 25 y 64 años alcanza al menos el nivel de secundaria y el $46.08 \%$ alcanza al menos el nivel de preuniversitario. El nivel promedio de los trabajadores es de $11^{\circ}$ grado (ONE, 2002). Son indicadores significativamente superiores a los de muchos países de la Unión Europea, y particularmente superiores a los de España. 
La totalidad de los residentes en edades de educación primaria tienen acceso a la educación primaria. Aun en los lugares de más difícil acceso por las condiciones geográficas, los niños residentes tienen escuela, en oportunidades con muy pocos alumnos, e incluso hay en el país varias escuelas con un solo alumno. Los alumnos enfermos o con discapacidades físicas reciben su educación en 422 escuelas especiales, en aulas hospitalarias o por maestros ambulatorios que les enseñan en sus propios domicilios. La tasa de matriculación en primaria asciende al $99.5 \%$. En el año 2005 existían en el país 9034 escuelas primarias.

Se dispone de las capacidades educativas necesarias para que todos los graduados de primaria accedan a la educación secundaria y continúen al nivel preuniversitario.

[...] Todo este esfuerzo, sin proponérnoslo, porque realmente no nos proponemos batir ningún record, ni establecer ninguna marca mundial, pero sin quererlo se ha establecido, Cuba tiene hoy un docente por cada 33.9 habitantes. ... de manera que Cuba hoy es el país con la mayor cantidad de maestros per cápita del mundo, gracias a todo este esfuerzo que ha hecho la Revolución [...] (GÓMEZ, 2006).

En el año 2005 existían en el país 1984 instituciones de nivel medio. (ONE, 2005)

Transformaciones de mucha trascendencia se han introducido en los últimos años en el nivel primario, disponiéndose actualmente de una enseñanza personalizada de mayor calidad, que comprende doble sesión diaria, con grupos de clase de 20 alumnos como máximo que utiliza la televisión y la computación como medios de enseñanza. Cada aula cuenta con televisor y reproductor de video y cada escuela cuenta con laboratorio de computación y una colección de clases grabadas en video por docentes de mucha experiencia y excelente preparación pedagógica y profesional. El país dispone de dos canales educativos con cobertura nacional, que trasmiten una programación diseñada para los distintos niveles de enseñanza ${ }^{3}$ que complementa la labor del maestro y los medios disponibles en el aula.

También en el nivel secundario se han introducido transformaciones importantes con una educación personalizada mediante grupos de 15 alumnos en aulas con televisor y reproductor de video, mediante un profesor general e integral que conduce el aprendizaje y que se auxilia con grabaciones de video y la programación de los canales educativos. Las

\footnotetext{
${ }^{3}$ En el año 2004 transmitieron 7576 horas y en el año 200510350 horas. (ONE, 2005)
} 
clases de idioma extranjero y educación física son impartidas por docentes especializados en estas materias.

En el nivel preuniversitario los grupos son de 30 alumnos y las clases son impartidas por docentes especializados en ciencias naturales, ciencias exactas, ciencias sociales y humanidades, idioma extranjero y educación física, pero el proceso de instrucción es complementado por un profesor responsable de la formación multilateral.

Los gastos públicos en educación fueron en el año 2004 de 3601.0 miles de pesos y en el año 20054273.3 miles de pesos (ONE, 2005), lo que representa un incremento del $19 \%$ de un año con respecto al otro.

\subsection{Indicadores de la educación superior}

El Censo Nacional de Población y Viviendas de Cuba (2002) reportó una población de 11,17 millones de habitantes, de los cuales 712672 son graduados universitarios, lo que significa que el $6.91 \%$ del total dispone de al menos un título de la educación superior, conjunto en el cual las mujeres constituyen la mayoría con un $52.05 \%$.

Son graduados universitarios el $11.63 \%$ del segmento de la población comprendido entre 30 y 59 años y el $10.01 \%$ de la Población Económicamente Activa, categoría esta última en la que el $10.45 \%$ son mujeres y el $8.30 \%$ son hombres. La Tasa Bruta de Matrícula ${ }^{4}$ en el curso académico 2006-2007 alcanza el 63.1\%.

La cobertura de la educación superior abarca toda la geografía del país, con la presencia de al menos cuatro instituciones de educación superior en todas las provincias y en el Municipio Especial Isla de la Juventud. En la provincia Habana existen 6, en la provincia Holguín 5 y en la capital 16 (ANEXO1).

Todos los residentes tienen la oportunidad de matricular cualquiera de las carreras de pregrado que se ofertan en el país, porque si las IES de su provincia no la ofertara el estudiante recibe de forma gratuita una beca que incluye residencia, manutención y gastos de bolsillos para cursarla en la provincia más cercana a su domicilio en la que se ofrezca la carrera de su preferencia.

Hay constituidas más de 3000 sedes universitarias municipales con cobertura en la totalidad de los 169 municipios del país.

El 65.16\% de la matrícula nacional de pregrado del curso 2005-2006 son mujeres. En las instituciones adscritas al MES son el $50.42 \%$, en las

\footnotetext{
${ }^{4}$ Por ciento de la matrícula total con respecto al segmento de la población comprendida entre 18 y 24 años.
} 
adscritas al MINED son el $71.27 \%$, en las adscritas al MINSAP son el $78.88 \%$, en el Instituto Superior de Arte son el 50.74\%, en la Educación a Distancia son el $54.00 \%$ y en las Sedes Universitarias Municipales son el $65.00 \%$. No son mayoría en la carrera de Cultura Física con el 29.09\%, en el Instituto de Diseño Industrial donde representan el 43.98\%, en el Instituto Superior de Ciencia y Tecnología Aplicada donde representan el 43.98\% ni en la Universidad de Ciencias Informáticas donde representan el $47.19 \%$.

\subsection{Transformación de la Educación Terciaria: la universalización de la educación superior (IESALC, 2004)}

Desde el curso académico 2001-2002 de la educación superior cubana se vienen produciendo nuevas e importantes transformaciones con vistas a ampliar las posibilidades de estudios superiores a los sectores sociales menos favorecidos, que forman parte del proceso único de gradual aproximación y perfeccionamiento hacia la universalización de la Universidad, con una visión más integral de los conceptos de equidad y justicia social, y sobre la base del concepto de acercar la Universidad hasta el lugar donde residen o trabajan las personas.

Surge una nueva cualidad de la Universidad, que radica en su presencia, cada vez con mayor intensidad, en todos los municipios del país. Decenas de miles de estudiantes provenientes de diferentes programas sociales, trabajadores de la industria azucarera y cuadros de organizaciones políticas y de masas, cursan estudios universitarios en sus municipios, localidades e incluso bateyes azucareros, en 43 carreras universitarias diferentes, que se imparten en más de 800 lugares diferentes.

Este proceso de ampliación de posibilidades de estudios superiores nunca antes visto, debe conducir a que, en un corto período de tiempo, todas las personas con título de acceso a la educación superior que deseen cursar estudios universitarios, tengan la posibilidad real de matricular alguna carrera.

La Universidad cubana, para responder a tales intenciones, realiza profundas transformaciones en sus concepciones académicas, que lejos de estar concluidas constituyen hoy punto de partida para premisas de las que deben producirse en lo adelante y que, en su integración deben caracterizar lo que se ha denominado Nuevo Modelo de Universidad, en respuesta a los actuales retos de la sociedad y en general a las transformaciones que tienen lugar en el mundo en la ciencia y en la tecnología. 
Esos retos y transformaciones conforman un escenario totalmente diferente al de etapas anteriores, caracterizado por un conjunto de características que hoy se abren paso con inusitada fuerza y por tanto requieren de una Universidad transformadora, flexible, dinámica, apta para brindar respuesta a las nuevas exigencias que tales circunstancias demandarán de la educación superior.

El hilo conductor fundamental de este conjunto de transformaciones que con un enfoque en sistema se acometen, lo constituye la Universalización de la educación Superior.

La Universalización caracteriza el sistemático proceso de transformaciones que ha tenido lugar en la educación superior, dirigido a la ampliación de posibilidades y oportunidades de acceso a la Universidad, con lo cual se contribuye a la formación de una cultura general integral de la población y a un incremento paulatino de mayores niveles de equidad y de justicia social en la sociedad.

En su etapa actual la Universalización garantiza un acelerado proceso de incorporación de jóvenes de familias de menor desarrollo cultural a los estudios universitarios, ampliando las oportunidades de esas familias para una integración más plena a la sociedad.

En correspondencia con ello, la Universalización de la educación superior hay que entenderla como un proceso que se inicia con el triunfo de la Revolución. Tiene como premisa la Campaña Nacional de Alfabetización -masivo proceso que involucró a miles de jóvenes en la noble tarea de enseñar a leer y escribir -y avanza y se fortalece gradualmente desde la Reforma Universitaria de 1962 hasta nuestros días.

La Reforma Universitaria fue proclamada el 10 de enero de 1962, estableciéndose la enseñanza universitaria gratuita y la creación de un sistema de becas que garantizó la posibilidad de estudios universitarios a estudiantes de los sectores más humildes de la población de todas las provincias del país y permitió establecer los nuevos compromisos que la enseñanza superior contraía con la sociedad. La ciencia y la técnica, en sus más elevadas manifestaciones, habrían de ponerse para siempre al servicio del pueblo y del desarrollo sostenido de la nación.

En este período se desarrollaron cursos de nivelación para decenas de miles de ciudadanos que no se habían podido formar como bachilleres, para de esta forma preparar a los futuros estudiantes que ingresarían a la Universidad.

Con el comienzo de los Cursos para Trabajadores a inicios de la década del 70, se produce un proceso de transformación dirigido a un nuevo incremento del acceso a la educación superior. Este significativo cambio 
en el ingreso fue denominado Universalización de la Universidad, en el que de forma masiva iniciaron estudios superiores trabajadores que tenían una titulación que les permitía acceder a la educación superior.

En el curso 1976-77 existía al menos una institución de educación superior en diez de las catorce provincias del país, con énfasis especial en las universidades médicas y universidades pedagógicas. Este es el período en que se crean las unidades docentes, para propiciar mayor integración de la docencia, la producción y la investigación en el nivel universitario, y se crean filiales y sedes universitarias.

En el mes de julio de 1976 se funda el Ministerio de Educación Superior y la red de centros con la responsabilidad de dirigir la política educacional en este nivel de enseñanza, imprimiéndole un decisivo impulso a los estudios universitarios en el país y al proceso de Universalización que demandó miles de docentes para cumplir con el incremento de la matrícula nacional, que en el curso 1975-1976 ascendía a 83957 estudiantes.

A finales de 1979 se inicia la Educación a Distancia que tiene como único requisito para su matrícula el decimosegundo grado vencido, con lo que se amplían más las fuentes y vías de acceso a los estudios universitarios. En los años 80 continúa creciendo la red de centros de educación superior y la matrícula universitaria alcanza su cifra histórica mayor en el curso 1987-88; la que ha sido superada con creces desde el curso académico 2004-2005. En la primera mitad de la década del 90 se acusa una reducción gradual de la matrícula de pregrado como consecuencia de la crisis derivada del derrumbe del socialismo europeo y la desaparición de la URSS 5 . En toda esa década se incrementa la educación de postgrado, en la que se

\footnotetext{
${ }^{5}$ El derrumbe del campo socialista y la posterior desaparición de la Unión de Repúblicas Socialistas Soviéticas (URSS) en 1991, representó para Cuba la pérdida súbita de sus principales mercados, así como de las favorables condiciones de precios y las ventajosas relaciones económicas que mantenía con esta comunidad de naciones. La capacidad de importación de más de 8000 millones de dólares anuales que el país alcanzó en 1989, se redujo en 1992 a 2200 millones y en 1993 a 1700 millones.

El descenso del Producto Interno Bruto (PIB) fue vertiginoso. Entre 1990 y 1993 el PIB decreció en un 34,8 \%. Los efectos del bloqueo norteamericano, que acumulan pérdidas directas e indirectas por más de 67000 millones de dólares en los últimos 40 años amortiguados durante mucho tiempo por las favorables condiciones de intercambio con el campo socialista, se hicieron repentinamente más agudos.

Las consecuencias de la recesión económica se reflejaron en el conjunto de la actividad social, pero se sostuvieron los beneficios de los servicios educacionales totalmente gratuitos, lográndose por la política aplicada que el costo de la crisis se compartiera entre todos y no se concentrara en los sectores de la población con menos ingresos.

Sin embargo, resultó imposible evitar que estos dramáticos acontecimientos desencadenados abruptamente y que conmovieron la estabilidad económica nacional, no tuvieran una determinada repercusión en la educación superior.
} 
superan y actualizan miles de graduados universitarios; asimismo, se atiende de forma priorizada la investigación científica en línea con el llamado del Presidente Fidel Castro de convertir a las universidades en centros de investigaciones.

En la actualidad tiene lugar una Nueva Etapa en la Universalización, cualitativamente superior, caracterizada por un franco proceso de despliegue que incluye, no solo las instalaciones universitarias tradicionales, sino también la incorporación de nuevas sedes y aulas universitarias en todos los municipios del país, permitiendo un acelerado incremento de nuevas fuentes de ingreso y tipos de cursos para estudios universitarios. Este desafío representa la más importante oportunidad, en toda la historia de la Revolución, para el desarrollo de las universidades en nuestro país.

Como parte de la Batalla de Ideas, la Dirección del País impulsó desde el año 2000 varios programas sociales vinculados con la educación superior, para lograr mayor equidad y justicia social en la sociedad cubana. Se convocó a la educación superior a garantizar la continuidad de estudios de los diferentes programas sociales creados primero en Ciudad de la Habana y Matanzas, y luego en todo el país.

Dentro de los conceptos que han revolucionado la educación superior cubana, en el contexto de la Universalización de la educación superior, el más trascendental es sin duda alguna el de llevar la Universidad a todos los municipios del país. Este propósito ya hecho realidad hoy en una primera fase de desarrollo, centrada en el proceso de formación, ha demandado de la educación superior la concepción y puesta en práctica de un Modelo Pedagógico propio para esta labor.

Ese modelo, constituye un importante pilar en esta concepción de la nueva Universidad cubana y debe integrarse a ella como una importante

A pesar de que la disminución del presupuesto asignado a la educación superior fue proporcionalmente menor que la del PIB expresión ésta de la alta prioridad que el Estado le concede a la educación, y en particular a la educación superior, como factor estratégico para resolver la crisis, por la caída de la capacidad de importación y su impacto en la economía nacional, los fondos para otros gastos e inversiones que se asignaban al sector, fueron recortados durante algunos años, sin que con ello se afectaran los salarios del personal ni los niveles básicos de beneficio de los estudiantes.

Los efectos del ajuste se hicieron sentir, entre otros, en el suministro de combustibles, energía eléctrica y equipos. Además, la súbita desaparición de mercados consolidados que amortiguaban el impacto del bloqueo norteamericano, dificultó durante varios años la obtención de insumos de baja significación financiera pero de amplio surtido que, como reactivos, materiales didácticos, de laboratorios y otros, se consumen en los procesos sustantivos de las IES y que tienen efectos perceptibles en la calidad de la educación. (CEPES, 2002; LÓPEZ SEGRERA, 1998) 
vía para la continuidad de estudios superiores, hasta ahora necesariamente limitada por razones materiales a determinadas fuentes de ingreso, provenientes fundamentalmente de algunos de los Programas Sociales, pero que gradualmente irá ampliándose a nuevos sectores sociales, en la medida en que las condiciones lo permitan y con ello se dispondrá de una importante forma de continuidad de estudios para jóvenes estudiantes egresados de preuniversitario y de institutos tecnológicos, trabajadores y en general personas que residan en esos territorios y que encuentran en esa modalidad la posibilidad de materializar sus aspiraciones de superación.

Esta nueva idea que se abre paso, lleva consigo una nueva cualidad, que se viene expresando en cada territorio y que consiste en que a partir de la creación de estas sedes o filiales universitarias, los municipios asumen un papel más activo en la gestión de los profesionales que necesitan para su desarrollo, para lo cual se requiere una alta integración de todos los factores, encabezados por el gobierno municipal. Solo de ese modo se lograrán cabalmente los ambiciosos objetivos que el país se ha propuesto con esta nueva etapa de la Universalización de la educación superior.

Las Sedes Universitarias Municipales, durante esta primera etapa de su desarrollo, han centrado su atención fundamentalmente en asegurar, con la calidad requerida, la continuidad de estudios de las diferentes carreras que en cada municipio se han comenzado a impartir. Esta ha sido una tarea de mucha envergadura y ha exigido de las sedes la completa dedicación a esta labor.

Unido a ello, y como parte de su propio desarrollo, las sedes han comenzado a dar los primeros pasos en la incorporación de otras acciones académicas propias de la vida universitaria, las que gradualmente se irán consolidando hasta lograr convertir a cada sede en una original universidad, en correspondencia con la hermosa aspiración de que la Universidad esté al alcance de todos los hombres y mujeres, cuestión que solo será posible lograr cuando la Universidad se haga presente, con todos sus procesos sustantivos, en cada uno de los municipios del país.

Lo anterior supone, en primer lugar, la ampliación gradual de la enseñanza de Cursos Regulares Diurnos en aquellas carreras en las que, por sus características, sea posible desarrollar el proceso de formación desde sus propios lugares de residencia. El crecimiento y fortalecimiento de las Unidades Docentes, la masiva incorporación de los profesionales del territorio a esta labor y el creciente apoyo que experimenta esta labor por las autoridades del Partido y el Gobierno en cada municipio, unido a niveles de integración superiores entre las diferentes expresiones de educación superior que allí existen, propiciará ir creando gradualmente 
las condiciones necesarias para que nuevas carreras, parcial o totalmente, se impartan en los diferentes municipios del país.

Algo similar se impone realizar en la concepción y desarrollo de los Cursos para Trabajadores. Nacidos como una conquista del movimiento obrero cubano, las Sedes Universitarias Municipales crean espacios en los que es posible organizar estudios de este tipo, en las carreras en que existan las condiciones para ello, evitando con esto que los trabajadores tengan que desplazarse a las cabeceras provinciales a participar en los encuentros que actualmente se ofrecen en las sedes centrales. La educación superior, en estos momentos prepara los fundamentos de un nuevo enfoque de estos cursos, bajo el cual será posible ofrecer, desde los municipios del país, algunas de las carreras de los Cursos Regulares para Trabajadores que hoy se ofrecen de forma concentrada en los centros de educación superior, dejando en estas instalaciones solo aquellas que son altamente demandadas lo que impide ofrecerla en los municipios.

El nuevo modelo de Universidad al que se convoca, en el que todas las personas con nivel medio superior interesadas en cursar estudios superiores tengan la posibilidad real de estudiar una carrera, eliminando todas las barreras que actualmente existen, se ha de sustentar en conceptos de esta naturaleza, que han de propiciar que amplios sectores sociales encuentren respuesta a sus necesidades de estudios superiores en las propias localidades donde residen, estudian o trabajan.

Una consecuencia natural del incremento de los niveles de estudios superiores en la sociedad ha de ser la de ofrecer, también desde los municipios, posibilidades de estudios posgraduados a aquellas personas que habiendo concluido sus estudios universitarios, requieren por su actividad laboral o lo desean como vía para su superación continua, realizar estudios de postgrado. Ya se dan los primeros pasos en esta dirección en algunas de las Sedes Universitarias municipales y este proceso se extenderá gradualmente hasta lograr que los estudios de postgrado se generalicen en todos los territorios del país.

Íntimamente ligado al desarrollo de los estudios de pregrado y postgrado en los diferentes municipios, y como vía para dar respuesta a problemas sociales, productivos o de los servicios, presentes actualmente en los diferentes territorios, la investigación científica se hará igualmente realidad en las Sedes Universitarias Municipales, cada vez con mayor fuerza y amplitud en la medida en que se eleven los niveles de calificación de las personas que allí residen. En particular las investigaciones de carácter social relacionadas con los principales problemas de la comunidad, encontrarán en las propias localidades el mejor laboratorio para el desarrollo 
de investigaciones de este tipo. De ese modo, los municipios estarán en condiciones no solo de formar los recursos humanos que se requiere para su desarrollo, sino también de utilizarlos para resolver los problemas de naturaleza científica que requieren de la investigación para ser resueltos.

Pero el papel de las Sedes Universitarias Municipales no sería todo lo amplia que pudiera ser si se limitara a los aspectos antes mencionados. La Universidad, además de preservar y desarrollar la cultura de la humanidad, tiene también la obligación de promoverla, de divulgarla, de hacer que llegue a todos los rincones de cada lugar. De ahí que en estrecha relación con los procesos antes descritos, la extensión universitaria tiene igualmente que hacerse presente en todas esas sedes con toda la amplitud que demanda una respuesta de este tipo, que no se limita solo a aquellos sectores que han tenido la oportunidad de alcanzar estudios universitarios, sino que debe llegar a cada una de las personas que residen en la comunidad y deseen elevar su nivel cultural. Los cursos de superación cultural general, las cátedras de la tercera edad, los proyectos de trabajo comunitario y otras formas del trabajo de extensión se desarrollarán con toda su amplitud en los territorios como expresión de la pujanza de la educación superior en cada uno de ellos.

\section{Educación Superior y Políticas Públicas de Ciencia y Tecnología (Política ..., 2006)}

Un primer período de "Promoción Dirigida de la Ciencia" se inscribe entre los años 1960 y 1977, con predominio del esquema de "Science Push". Es una etapa identificada por las transformaciones revolucionarias, el acceso masivo a la educación, la creación de un sistema nacional de salud, en la que se inicia y escala el bloqueo de los Estados Unidos. Entre los años 1960 y 1970 se crearon siete instituciones científicas por año como promedio. La educación superior institucionaliza las carreras de ciencias e ingenierías y crea una agenda de investigación centrada en problemas económicos y sociales. Comienza la formación masiva de recursos humanos de alto nivel aprovechando la cooperación internacional. Aparecen instituciones de coordinación de la ciencia y la tecnología, en 1974 el Consejo Nacional de Ciencia y Técnica y en 1976 el Comité Estatal de Ciencia y Técnica.

Le sucede un segundo período de "Modelo de Dirección Centralizada" hasta 1990, caracterizada por la concentración de la cooperación y el comercio con los países socialistas de Europa, como consecuencia de la incorporación de Cuba en 1972 al Consejo de Ayuda Mutua Económica 
(CAME). Es dominante la transferencia de tecnología y se potencia una mejor utilización de los resultados, incorporándolos a la producción y los servicios. A partir de la mitad de la década del ochenta la ciencia se organiza a través de Programas Científico Técnicos con diferentes alcances: nacionales, ramales y territoriales. Aparece en 1987 el Sistema de Introducción de Logros, se duplica el número de investigadores y se crea un sistema nacional de grados científicos.

Como consecuencia del derrumbe del socialismo europeo y la desaparición de la URSS y del CAME, el país introdujo cambios en el modelo económico y se ha visto obligado a producir un importante proceso de reconversión en la agricultura y la industria. Un significativo auge de los servicios de turismo se ha experimentado paulatinamente desde entonces y nuevos productos del conocimiento tienen cada vez más importancia entre las exportaciones del país.

En este contexto un nuevo período de "Modelo de Ciencia e Innovación Tecnológica" se ha ido consolidando en la dirección y organización de la ciencia y la tecnología. La creación del Polo Científico-Productivo del Oeste de La Habana y de cada provincia del país, consagrados a la Biotecnología y a la Industria Médico Farmacéutica; la promoción de movimientos sociales que impulsan y valorizan la innovación (Forum de Ciencia y Técnica, Asociación de Innovadores y Racionalizadotes, Brigadas Técnicas Juveniles); un mayor énfasis de la gestión tecnológica en las empresas; creación de grupos de investigación en las universidades que trabajan a ciclo completo; la ampliación y endogenización de la educación de postgrado; la municipalización de la educación superior que propicia nuevas oportunidades a la innovación local; la promoción de la suficiencia alimentaria y la agricultura sostenible; y la aparición de un sector de Economía del Conocimiento a partir de la Biotecnología y los avances en la Industria del Software; constituyen los principales rasgos de este modelo.

\section{Análisis del Sistema de Oferta de Educación Terciaria}

\subsection{Tipología de las IES y Tipos de Cursos}

Todos los servicios educativos de Cuba son de carácter público y se ofrecen de manera gratuita, incluyendo los de educación superior que comprende también el nivel de postgrado.

El Sistema Nacional de Educación está integrado por siete subsistemas: Educación Preescolar, Educación General Politécnica y Laboral, Educación Especial, Educación Técnica y Profesional, Formación 
y Perfeccionamiento de Personal Pedagógico y Educación de Adultos y Educación Superior.

Corresponde al Ministerio de Educación Superior la dirección metodológica del subsistema de Educación Superior, pero también a otros Organismos de la Administración Central del Estado (OACE) se adscriben instituciones de educación superior (IES); así, los Institutos Superiores de Ciencias Médicas se adscriben al Ministerio de Salud Pública; los Institutos Superiores Pedagógicos al Ministerio de Educación; el Instituto Superior de Cultura Física al Instituto Nacional de Deportes Educación Física y Recreación; el Instituto Superior de Relaciones Internacionales al Ministerio de Relaciones Exteriores; el Instituto Superior de Arte al Ministerio de Cultura; el Instituto Superior de Diseño Industrial al Ministerio de Economía y Planificación; el Instituto Superior de Tecnologías y Ciencias Aplicadas al Ministerio de Ciencia, Tecnología y Medio Ambiente; la Universidad de las Ciencias Informáticas al Ministerio de la Informática y las Comunicaciones; y los Institutos, Academias y Escuelas Militares Superiores al Ministerio de las Fuerzas Armadas Revolucionarias y al Ministerio del Interior.

Al Ministerio de Educación Superior se adscriben directamente las IES cuyos campos de estudio son las Ciencias Naturales y Matemática, Ciencias Sociales y Humanísticas, Ciencias Económicas y Contables, Ciencias Técnicas y Ciencias Agropecuarias.

La red de educación superior de la República de Cuba dispone de 65 instituciones y 3150 sedes universitarias municipales.

Tipología de las IES cubanas (CEPES, 2002).

\section{Universidad:}

Formación de profesionales en diferentes áreas del saber, tales como en las ciencias: naturales y matemática, sociales y humanísticas, económicas, técnicas y agropecuarias. En la institución pueden desarrollarse parte o la totalidad de las áreas antes relacionadas.

Instituto Superior Politécnico:

Se encarga de la formación de profesionales en el campo de las ciencias técnicas y arquitectura, para varias ramas de la economía nacional.

\section{Instituto Superior:}

Encargado de la formación de profesionales con mayor peso en algunas de las áreas del saber. Los más representados en la red son: los de ciencias médicas; ciencias pedagógicas y el de cultura física y deportes. En ocasio- 
nes estas instituciones son denominadas como Escuela o Academia, en la mayoría de estos casos su área de conocimiento son las ciencias militares.

\section{Centro Universitario:}

Consiste en una etapa organizativa transitoria hasta alcanzar las condiciones objetivas y subjetivas que permitan pasar a uno de los tipos de instituciones antes referidas.

\section{Filial Universitaria:}

Tiene un carácter dependiente a otra IES y por los niveles de servicios que debe prestar no necesariamente debe evolucionar hacia otro tipo de institución.

\section{Sede Universitaria Municipal:}

Orientada al desarrollo de actividades propias de la educación superior en los territorios. Tiene un carácter dependiente de una IES determinada. Durante una primera etapa de su desarrollo, han centrado su atención fundamentalmente en asegurar, con la calidad requerida, la continuidad de estudios de las diferentes carreras que en cada municipio se ofrecen. Han comenzado a dar los primeros pasos en la incorporación de otras acciones académicas propias de la vida universitaria, asumiendo funciones también de investigación, docencia de postgrado y extensión universitaria.

\section{Escuela Latinoamericana o Escuela Internacional:}

Existen dos instituciones de este tipo, una para el área de medicina y la otra para la educación física y el deporte. Su misión se orienta a la formación de estudiantes extranjeros en la condición de becarios, por lo que los gastos de preparación los asume el Estado cubano desde una perspectiva de colaboración solidaria con otros países en vías de desarrollo. En estas IES se encuentran también estudiantes cubanos en menores magnitudes. En el orden administrativo tienen un carácter independiente y en los aspectos académicos se subordinan a un instituto superior de esa Rama

\section{Facultad Independiente de Ciencias Médicas:}

Son entidades que integran la red de su Rama, con similares responsabilidades y atribuciones a los institutos superiores, tomando en consideración las necesidades territoriales para la formación e igualmente relacionadas con los servicios de salud pública de sus áreas de influencia. En el orden administrativo tienen un carácter independiente y en los aspectos académicos se subordinan a un instituto superior de esa Rama.

Las IES ofrecen cursos de formación de pregrado o licenciatura, cuyo nivel se corresponde con el descrito por el nivel 5 A de la Clasificación 
Internacional Normalizada de la Educación (CINE); y de formación de postgrado que se corresponde con el nivel 6 de la citada clasificación. En algunas instituciones que han adquirido un importante grado de desarrollo y madurez, las ofertas de postgrado tienen mayor peso que las de pregrado

El subsistema de Educación Superior cubano no dispone de instituciones postsecundarias no universitarias.

Tipos de cursos (CEPES, 2002):

Cursos regulares diurnos:

Con dedicación a tiempo completo al que acceden principalmente los jóvenes que culminan el nivel medio superior (bachillerato) y cumplen los requisitos que se establecen para la carrera de que se trate.

\section{Cursos regulares para trabajadores:}

Con dedicación a tiempo parcial, se ofertan a trabajadores que mantienen su vínculo laboral mientras estudian. Pueden ser vespertinonocturnos o por encuentros ${ }^{6}$

\section{Cursos a distancia:}

El estudiante recibe apoyo mediante guías de estudio, textos y páginas Web, además de consultas presenciales con profesores. El acceso es irrestricto. En la actualidad esta modalidad se amplía con la oferta de nuevos productos multimedia.

\section{Cursos de continuación de estudios:}

Se desarrolla en las sedes universitarias municipales. La modalidad de estudio es semipresencial y el modelo pedagógico se caracteriza por su flexibilidad, para adaptarse a diversas situaciones laborales, a particularidades territoriales y al ritmo individual de aprovechamiento académico del estudiante. (LA UNIVERSIDADE..., 2006)

\subsection{Dimensión del sistema de educación terciaria.}

El subsistema de educación superior cubano está integrado por 65 instituciones de educación superior y 3150 sedes universitarias municipales. La matrícula estimada para el curso 2006-2007 sobrepasa los 620000 estudiantes.

\footnotetext{
${ }^{6}$ Los cursos vespertino-nocturnos tienen definidas actividades de clase en horarios fuera de la jornada laboral con dos o tres frecuencias a la semana. Por lo general, los cursos por encuentros desarrollan las actividades de clase cada dos semanas en días no laborables.
} 
La evolución del tamaño del subsistema durante los últimos 30 años se puede apreciar en el gráfico que se muestra a continuación:

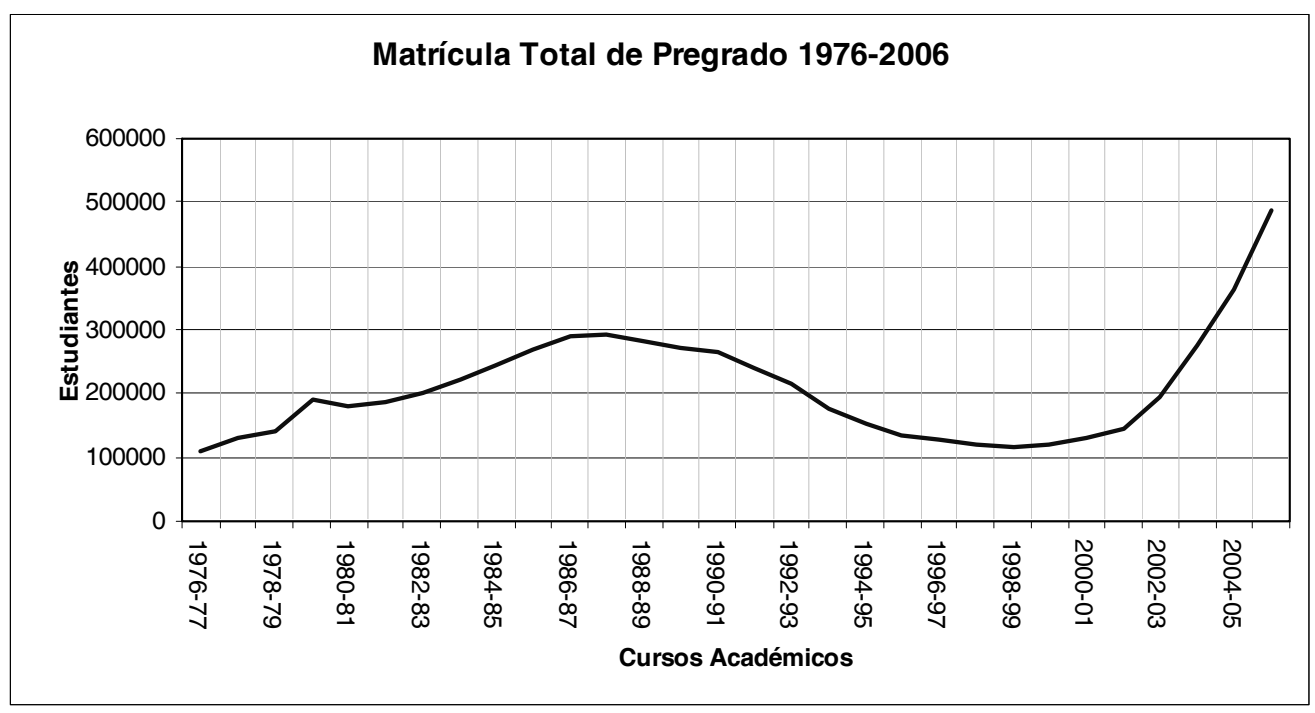

Se mantuvo un crecimiento sostenido hasta el curso 1987-1988 en que alcanzó el máximo histórico con 293722 estudiantes. A partir del curso siguiente se produjo una disminución paulatina como consecuencia de cambios en la composición demográfica por edades de la población primero, y como secuela de la crisis económica después, hasta que en el curso académico 2000-2001 comienza nuevamente a crecer, rebasa el máximo histórico alcanzado hasta ese momento en el curso académico 2004-2005 y continúa creciendo en la actualidad con incrementos promedio anual en los últimos cinco años superiores al $30 \%$.

Se experimentan incrementos en la matrícula de todas las ramas de la ciencia, excepto en las Ciencias Naturales y Exactas. Los mayores incrementos se producen en Ciencias Sociales y Humanísticas, en Ciencias Médicas, en Cultura Física y en Ciencias Económicas.

4.3 Títulos que otorga el sistema de educación terciaria (IESALC, 2005)

Las titulaciones ofrecidas por la educación superior cubana son las siguientes:

\section{Estudios de pregrado:}

\section{Licenciado:}

Carreras para el servicio social que tienen una extensión por lo regular de 5 años. Este título lo obtienen los graduados en Ciencias Naturales y 
Matemática, Económicas, Sociales y Humanísticas, Pedagógicas, Artes y Cultura Física. En las Ciencias Médicas los graduados de Enfermería, Tecnología de la Salud y Psicología de la Salud.; y en las Ciencias Técnicas los graduados de Diseño Industrial y de Diseño Informacional.

\section{Ingeniero:}

Carreras de aplicación en la producción y los servicios que tienen una extensión por lo regular de 5 años. Este título lo obtienen los graduados de Ciencias Técnicas y de Ciencias Agropecuarias.

\section{Doctor:}

Lo obtienen los graduados de Ciencias Médicas que tiene una duración de 6 años y los graduados de Ciencias Veterinarias que tiene una duración de 5 años.

\section{Arquitecto:}

Lo obtienen los graduados de Arquitectura que tiene una duración de 5 años.

La matrícula nacional de pregrado para el curso 2005-2006 es de 620000 estudiantes y hasta julio de 2006 el total de graduados sobrepasaba la cifra de 800000 .

\section{Estudios de postgrado:}

Se corresponden con el nivel 6 de la Clasificación Internacional Normalizada de la Educación (CINE). Son estudios avanzados en investigaciones originales y no están basados únicamente en cursos. Se estructura en dos variantes principales: superación profesional y formación académica.

La superación profesional tiene como objetivo la formación permanente y la actualización sistemática de los graduados universitarios, el perfeccionamiento del desempeño de sus actividades profesionales y académicas, así como el enriquecimiento de su acervo cultural, cuyas formas organizativas principales son el curso, el entrenamiento y el diplomado.

La formación académica tiene como objetivo la educación posgraduada con una alta competencia profesional y avanzadas capacidades para la investigación y la innovación, lo que se reconoce con un título académico o un grado científico. Sus formas organizativas son la especialidad, la maestría y el doctorado. 
Las certificaciones, títulos y grados que se otorgan en el postgrado son los siguientes: Certificado de Curso de Postgrado, Certificado de Entrenamiento de Postgrado, Certificado de Diplomado, Título Académico de Máster, Título Académico de Especialista, Grado Científico de Doctor de Determinada Ciencia y Grado Científico de Doctor en Ciencias.

\section{Curso de Postgrado:}

Posibilita la formación básica y especializada de los graduados universitarios; comprende la organización de un conjunto de contenidos que abordan resultados de investigación relevantes o asuntos trascendentes con el propósito de complementar o actualizar los conocimientos de los profesionales que los reciben. Tienen una extensión mínima de dos créditos.

\section{Entrenamiento de Postgrado:}

Posibilita la formación básica y especializada de los graduados universitarios, particularmente en la adquisición de habilidades y destrezas y en la asimilación e introducción de nuevos procedimientos y tecnologías con el propósito de complementar actualizar, perfeccionar y consolidar conocimientos y habilidades prácticas. Tiene una extensión mínima de dos créditos.

\section{Diplomado:}

Tiene como objetivo la especialización en un área particular del desempeño, y propicia la adquisición de conocimientos y habilidades académicas, científicas y/o profesionales en cualquier etapa del desarrollo de un graduado universitario de acuerdo con las necesidades de su formación profesional o cultural. Está compuesto por un sistema de cursos y/o entrenamientos y otras formas articuladas entre sí, que culmina con la realización y defensa de un trabajo ante tribunal. La extensión mínima es de 15 créditos.

\section{Maestría:}

Es un programa de formación académica posgraduada que proporciona a los graduados universitarios una amplia cultura científica y conocimientos avanzados en las áreas del saber, una mayor capacidad para la actividad docente, científica, la innovación o la creación artística, en correspondencia con las necesidades del desarrollo económico, social y cultural del país.

\section{Especialidad de Postgrado:}

Tiene como objetivo capacitar al egresado de nivel superior técnica y profesionalmente, de manera sistematizada para que profundice en el 
dominio integral de conocimientos, técnicas, métodos y procedimientos de desempeño de la misma actividad profesional que realiza, y puede implicar también la actualización de parte del personal calificado ante la introducción de tecnologías novedosas para el desempeño laboral en determinados puestos de trabajo ya existentes.

\section{Doctorado en Ciencias de Determinada Especialidad:}

Graduados de nivel universitario que contribuyan significativamente al desarrollo de su especialidad y satisfagan a plenitud los requisitos y las evaluaciones correspondientes a los programas que se establezcan, dentro de un proceso que culminará con la defensa ante un tribunal competente de una tesis donde se ponga de manifiesto un determinado grado de madurez científica, su capacidad de enfrentar y resolver problemas científicos de manera independiente, se demuestre un profundo dominio teórico y práctico en el campo del conocimiento de que se trate, y que contenga la exposición del resultado alcanzado por el graduado universitario en su especialidad, que podrá consistir en la propuesta de solución o solución de un problema teórico o práctico de ella o en una contribución científica de otro tipo.

\section{Doctorado en Ciencias:}

Doctores en Ciencias de Determinada Especialidad que hayan realizado un trabajo de alto nivel de especialización en el campo del conocimiento al que se dediquen, con la defensa ante un tribunal competente de una tesis que contenga la solución y generalización de un problema de carácter científico que constituya un aporte a la rama del conocimiento de que se trate.

El total de participantes en actividades de postgrado al cierre de diciembre de 2005 alcanzaba la cifra de 599405 personas, 76870 en "Cursos", 20124 en "Entrenamientos", 76870 en "Diplomados", 98795 en "Maestrías", 14296 en "Especialidades de Postgrado" y 4129 aspiraban al grado de Doctor. Hasta julio de 2006, en el sistema nacional de postgrado, más de 16300 se habían titulado como Máster, 45000 habían alcanzado el título de Especialista y más de 7300 habían obtenido el grado de Doctor.

\subsection{Aportes del sistema de educación terciaria al desarrollo del país}

La educación superior cubana ha satisfecho las principales necesidades de profesionales que el país demanda para sus necesidades productivas, de servicios e investigativas, así como para cumplir eficazmente con sus 
compromisos de ayuda solidaria con otros países del mundo, particularmente en educación y salud pública.

La educación superior ha formado suficientes médicos y otros especialistas de la salud que le permiten al país disponer de un sistema de salud pública universal, gratuita y accesible a toda la población. El sistema de salud pública está regionalizado y tiene un carácter integral, al alcance de todos los ciudadanos en el campo y en la ciudad. El bajo índice de mortalidad infantil logrado es el menor de la Región y está entre los mejores del mundo. Cuba dispone del mayor índice de médicos por cada mil habitantes del mundo. Actualmente más de 20000 médicos cubanos brindan servicios en más de sesenta diferentes países. Cuba ofrece miles de becas gratuitas para estudiantes de países del tercer mundo para formarse como graduados universitarios en diferentes ramas de la ciencia, particularmente en medicina se forman actualmente más 12000 .

El subsistema de educación terciaria dota al sistema educativo de los docentes que demanda, incluyendo a los que con carácter emergente ha necesitado para afrontar las transformaciones que se han producido en los últimos años en los distintos niveles educativos, y les ofrece las condiciones necesarias para su superación permanente; más del $90 \%$ son graduados universitarios y los que no lo son estudian para alcanzar este nivel en aulas universitarias. Actualmente más de 70000 maestros siguen cursos de maestría.

La formación de un número importante de profesionales con alta calificación en diversas ramas de la ciencia y con vocación social, ha sustentado las estrategias de desarrollo económico y social adoptadas por Cuba en los últimos años, lo que le ha permitido superar con éxito las principales dificultades de la crisis económica de los años noventa y sentar sólidas bases para alcanzar nuevos y mayores resultados.

Matrícula Nacional de Postgrado año 2005.

\begin{tabular}{|c|r|r|r|r|r|}
\hline Postgrado & \multicolumn{1}{|c|}{ TOTAL } & \multicolumn{1}{c|}{ MES } & \multicolumn{1}{c|}{ MINED } & MINSAP & \multicolumn{1}{c|}{ OTROS } \\
\hline Cursos & 385191 & 108629 & 106044 & 115289 & 55229 \\
\hline Diplomados & 76870 & 20706 & 33030 & 15578 & 7556 \\
\hline Entrenamientos & 20124 & 5360 & 2605 & 7127 & 5032 \\
\hline Maestría & 98795 & 10617 & 76455 & 10833 & 890 \\
\hline Especialidad & 14296 & 2564 & 91 & 11457 & 184 \\
\hline Doctorado & 4129 & 2145 & 965 & 452 & 567 \\
\hline TOTALES & 599405 & 150021 & 219190 & 160736 & 69458 \\
\hline
\end{tabular}

Fuente: Departamento de Estadísticas del MES (2005). 


\section{Aspectos Estratégicos de la Educación Terciaria}

\subsection{Educación terciaria, economía y empleo}

Una de las vías más relevantes de esta relación con el sistema económico son las unidades organizativas denominadas Unidades Docentes y Entidades Laborales Base. Las primeras son instituciones laborales representativas y de avanzada del sistema productivo y de servicios, donde existen condiciones para lograr la expresión máxima de integración de la docencia-producción- investigación?extensión; y las segundas son centros laborales, donde, en general, hay condiciones para realizar, fundamentalmente, las prácticas profesionales de los estudiantes. Esta cooperación se desarrolla sobre la base de convenios y planes de trabajo entre las IES y las entidades de producción y servicios, que establecen compromisos en el pregrado, el postgrado (programas de educación continua), la investigación, y la extensión universitaria.

El balance de la cooperación es valorado en todas las IES y Organismos de la Administración Central del Estado (OACE) y sus entidades laborales como estratégico y positivo. Entre los aspectos generales más relevantes, que se señalan como resultado de la referida cooperación, son los siguientes:

a) Acciones conjuntas para el establecimiento, desarrollo y consolidación de la red de Unidades Docentes y Entidades Laborales Bases;

b) Avances en la categorización docente de los profesionales de la producción y los servicios, que participan en el desarrollo de actividades docentes e investigativas;

c) Desarrollo de múltiples cursos de capacitación y de diferentes modalidades de postgrado (educación continua para el mejoramiento profesional y humano de los profesionales desde su puesto de trabajo);

d) Participación conjunta en investigaciones e introducción de resultados tomando en cuenta los avances de la ciencia y la tecnología;

e) Participación conjunta en proyectos comunitarios y en diferentes acciones de extensión universitaria, orientados a elevar la cultura general integral de la población;

f) Aportes materiales y financieros de los OACE para fortalecer, sobre todo el área del pregrado, y también del postgrado y la investigación. 
En la educación de pregrado, es de destacar que en todas las carreras que se desarrollan en los cursos regulares diurnos, el proceso formativo contempla la vinculación de los estudiantes con el trabajo en su profesión en diferentes entidades de la producción y los servicios. De esta manera las IES garantizan la formación de recursos humanos de nivel superior que puedan integrarse con rapidez y éxito en el sector productivo y de servicios, mediante su preparación en las condiciones reales de su ejercicio profesional.

En el sistema de educación de postgrado, sus dos subsistemas (la superación profesional y la formación académica) permiten atender las necesidades del sistema económico, además de las de superación personal, así como establece relaciones estables entre IES y empresas u otros sectores importantes de la vida social.

Para dar respuesta a las necesidades del sistema económico, el postgrado toma en cuenta la existencia de tres escenarios: el de los sistemas de los ministerios y organismos, el regional y el de la actividad posgraduada de carácter más académico.

El vínculo permanente de las IES con las empresas y demás organismos, garantiza que la elevación de la capacitación del cuerpo docente se revierta en beneficio de otras esferas de la sociedad. Atendiendo a las necesidades de superación de los profesionales que laboran en las áreas de la producción o los servicios, las instituciones establecen convenios de colaboración con empresas, organismos y gobiernos de los territorios donde están enclavadas.

La alianza entre las empresas y las IES ha permitido también la creación de programas de formación a nivel de maestría, especialidad y doctorado, a partir de la determinación de las necesidades de superación de los profesionales y la consecuente formación académica de éstos. En ocasiones, si una sola institución no es suficiente para la atención de tales necesidades, se coordinan las acciones estableciendo una red entre diversas IES a partir de la solicitud del organismo correspondiente.

Los resultados de las investigaciones que se realizan en el Subsistema, tienen un importante impacto en el desarrollo del sistema económico y el empleo.

Un papel importante en la relación con el sistema económico han sido las entidades de interfaz creadas en las IES, para acelerar la transferencia de resultados a las entidades de producción y servicios. Éstas han permitido elevar la eficacia de la transferencia de resultados y de la obtención de recursos financieros y materiales para las IES. 


\subsection{Educación terciaria e institucionalidad democrática}

La alta tasa de matrícula universitaria de más del $60 \%$ es un indicador del proceso de consolidación de una democracia económica, social y política. El bloqueo de EE.UU., que impone una situación permanente de acoso a la Isla, obstruye un mayor desarrollo de sus instituciones políticas, dado que se vive en una situación de emergencia nacional.

La entrada en vigor de la Constitución socialista en 1976 marcó un hito esencial en la historia política y jurídica contemporánea de Cuba y, por supuesto, en su proceso institucionalizador. No sólo consagra en su parte dogmática las grandes conquistas sociales, políticas y económicas logradas durante los años del poder revolucionario, sino que articula, en su parte orgánica, al nuevo aparato del poder -El Poder Popular.

El desafío más importante es lograr el perfeccionamiento del sistema jurídico y la concepción de democracia socialista en una situación de hostilidad permanente por parte de los sucesivos gobiernos de EE.UU. norteamericanos.

\subsection{Educación terciaria y políticas públicas de salud y educación}

La normativa actual que rige la estructura, funciones, atribuciones y el desempeño de la educación superior como un Subsistema, parte, en primer lugar de la Constitución de la República de Cuba de 1976, con las modificaciones realizadas en 1992.

Así, el Capitulo V de la Constitución referido a la "Educación y Cultura", responsabiliza al Estado Cubano con la orientación, fomento y promoción de la educación, la cultura y las ciencias en todas sus manifestaciones. Destaca que la política educacional se basa en los avances de la ciencia y la técnica, el ideario martiano y la tradición pedagógica cubana y universal. Consagra que la enseñanza es función del Estado y es gratuita, mantiene un amplio sistema de becas y proporciona diversas facilidades de estudio a los trabajadores sin distinción, para que puedan alcanzar los más altos niveles en el conocimiento científico técnico.

La Constitución precisa la integración y estructura del sistema nacional de enseñanza, así como la obligatoriedad de estudios definida como la preparación general básica, mínima para todos los ciudadanos. Además, promueve la educación patriótica y consagra la libertad en la actividad creadora e investigativa en la ciencia y prioriza en ésta la dirigida a resolver los problemas que atañen al interés de la sociedad y al beneficio del pueblo. 
Entre otros postulados se señala la responsabilidad de la familia, la escuela, los órganos estatales y las organizaciones de masas y sociales como las responsables de la atención a la formación integral de la niñez y la juventud.

En el Capitulo VI "Igualdad", se expresa textualmente en el articulo 42: "La discriminación por motivo de raza, color de la piel, sexo, origen nacional, creencias religiosas y cualquier otra lesiva a la dignidad humana está proscrita y es sancionada por la ley". "Las instituciones del Estado educan a todos desde la más temprana edad, en el principio de la igualdad de los seres humanos".

Más adelante, el articulo 51 del Capitulo VII "Derechos, Deberes y Garantías Fundamentales", consagra como un derecho ciudadano, dígase derecho humano, el derecho a la educación al expresar:

Todos tienen derecho a la educación. Este derecho está garantizado por el amplio y gratuito sistema de escuelas, seminternados, internados y becas, en todos los tipos y niveles de enseñanza, y por la gratuidad del material escolar, lo que proporciona a cada niño y joven, cualquiera que sea la situación económica de su familia, la oportunidad de cursar estudios de acuerdo con sus aptitudes, las exigencias sociales y las necesidades del desarrollo económico social.

Los hombres y mujeres tienen asegurado este desarrollo, en las mismas condiciones de gratuidad y con facilidades especificas que la ley regula, mediante la educación de adultos, la enseñanza técnica y profesional, la capacitación laboral en empresas y organismos del Estado y los cursos de educación superior para los trabajadores.

En este artículo se reflejan los derechos fundamentales para la educación de los ciudadanos cubanos, de su interpretación podrá comprenderse gran parte de la política del Estado en materia de educación como un bien público, para todos y en los niveles educacionales.

Solo un estado comprometido en el cumplimiento de una educación terciaria y políticas públicas de salud y educación que implican elevadas inversiones en capital social y humano - cobertura universal gratuita tanto en la educación como la salud - puede obtener los resultados alcanzados por el país en la actividad deportiva internacional a partir de una participación masiva en la cultura física y el deporte.

Los principales retos que enfrenta hoy el sistema de salud y educación de Cuba obedecen a falta de recursos materiales debido a las deformaciones producidas por el colonialismo primero y el neocolonialismo después, a lo que se añaden las inmensas pérdidas económicas por el bloqueo económico 
del gobierno de EE.UU. y catástrofes climáticas. Pese a esto, Cuba ha logrado mantener y desarrollar los sistemas de salud y educación - pese a un cierto estancamiento que se observó en los 90s al tener que reorientar su colaboración económica debido al derrumbe de la entonces URSS y de los países socialistas de Europa del Este - y mantener la cobertura universal gratuita.

La investigación médica, la cultura física y los deportes y los procesos de evaluación y acreditación de la educación superior - así como las políticas educativas- tienen en Cuba el nivel de los países desarrollados, por esa razón son áreas de interés para intensificar la colaboración con España.

\subsection{Acceso y equidad}

En Cuba el acceso es un proceso de carácter democrático por su gratuidad e igualdad de oportunidades a todos los aspirantes, con la única limitación de sus capacidades.

Facilitan el acceso - para fortalecer la equidad y evitar la inmigración interior y la concentración en grandes núcleos ? la existencia de tres modalidades de cursos: los Cursos Regulares Diurnos (CRD) con dedicación a tiempo completo, los Cursos Regulares para Trabajadores (CPT) con dedicación a tiempo parcial, que permiten mantener un vínculo laboral y los Cursos a Distancia.

Existen otras vías de acceso específicas en atención a determinados intereses sociales, entre ellas, para los jóvenes residentes de las zonas montañosas del país se define un plan de plazas que les permite cursar carreras del perfil agropecuario en las facultades creadas en esos territorios, lo que no limita a que puedan aspirar a las diversas carreras que se imparten en otras IES. También para los atletas que integran los equipos deportivos de mayor nivel en el país se les ofrece de manera diferenciada un plan de plazas en las diferentes carreras. En todos los casos el proceso para otorgar la plaza se basa en un escalafón a partir del rendimiento académico de los aspirantes.

La política vigente establece el compromiso de garantizar un puesto de trabajo al graduado de los cursos regulares diurnos (CRD), es por ello, que para cada año académico se aprueba un plan de plazas para cada provincia, que tiene en cuenta las necesidades de fuerza de trabajo calificada según la demanda determinada territorialmente, proceso que es dirigido por el Ministerio de Economía y Planificación con la participación de las IES, las entidades laborales y Organismos de la Administración Central del Estado (OACE). En la base del análisis, se encuentran las capacidades 
de las IES y la población de estudiantes que culminarán el grado 12 (preuniversitario).

En relación a las características de los exámenes de ingreso en los CRD se establece que el aspirante debe examinar determinadas asignaturas y/o aprobar pruebas de aptitudes, según las carreras por las que opta.

Como parte de la política de favorecer aún más el desarrollo educacional y cultural de toda la población, de la lucha por eliminar los focos de marginalidad en las grandes ciudades, y en el empeño de alcanzar una mayor justicia social, se inician a partir del año académico 2000-2001 nuevas acciones orientadas a fortalecer la atención social en las grandes ciudades del país, experiencia iniciada en Ciudad de La Habana, con el diseño de cursos para formar trabajadores sociales a partir de jóvenes graduados de grado 12, no vinculados eventualmente al estudio. A estos jóvenes, una vez graduados, se les facilita el acceso a la educación superior en un grupo de carreras de Ciencias Sociales y Humanísticas, combinando el trabajo con el estudio a distancia asistido por profesores. Esto ha dado lugar a la universalización de la universidad y a un incremento sustancial en la tasa de matrícula universitaria que pasó del $20 \%$ al $63 \%$ entre el 2002 y el 2006.

La Educación a Distancia es otra vía de acceso que, junto a la presencia de IES en las distintas provincias y a la universalización de la universidad, contribuye al desarrollo territorial y evita la inmigración interior masiva y la concentración en mega-universidades de grandes núcleos de estudiantes. A la educación a distancia accede cualquier ciudadano interesado, con el único requisito de poseer la certificación de graduado de nivel medio superior.

En resumen, para la política de la educación superior cubana, el proceso de acceso ocupa un espacio de alta prioridad en el empeño de que el mérito académico no sea el único factor que se tome en consideración, sino que deben adoptarse medidas que favorezcan la equidad. Es por ello que los rasgos que han madurado en el modelo de acceso, a lo largo de la década de los años 90 y primeros años del siglo XXI, se resumen en: a) la flexibilidad de las diferentes vías que permiten una mejor conciliación con las características, intereses y posibilidades del aspirante; b) el ofrecer diversas oportunidades para ejercer el derecho al acceso a estudios superiores desconcentrando demográficamente en las diversas provincias y municipios las posibilidades de estudio de diversas carreras. 


\section{ANEXOS}

\section{ANEXO 1: Red de IES de Cuba:}

Provincia de Pinar del Río:

Universidad de Pinar del Río "Hermanos Saíz Montes de Oca (UPR)

Instituto Superior Pedagógico "Capitán San Luis"

Facultad de Ciencias Médicas de Pinar del Río

Facultad de Cultura Física de Pinar del Río

Provincia Ciudad de La Habana:

Universidad de La Habana (UH)

Instituto Superior Politécnico "José Antonio Echeverría" (ISPJAE)

Instituto Superior Pedagógico "Enrique José Varona"

Instituto Superior de Ciencias Médicas de La Habana

Instituto Superior de Relaciones Internacionales "Raúl Roa García" (ISRRI)

Instituto Superior de Arte (ISA)

Instituto Superior de Diseño Industrial (ISDI)

Instituto Superior de Tecnologías y Ciencias Aplicadas (INSTEC)

Instituto Superior Pedagógico de Enseñanza Técnica y Profesional "Héctor A. Pineda"

Instituto de Cultura Física "Manuel Fajardo" (ISCFMF) ${ }^{7}$

Instituto Superior del MININT "Eliseo Reyes Rodríguez - Capitán San Luis"

Instituto Superior de Medicina Militar "D. Luis Díaz Soto"

Instituto Técnico Militar "José Martí"

Academia de las Fuerzas Armadas Revolucionarias "General Máximo Gómez"

Escuela Militar Superior "Arides Estévez"

Escuela Superior del Partido Comunista de Cuba "Ñico López"

Escuela Latinoamericana de Medicina (ELAM)

Escuela Nacional de Tropas Especiales "Baraguá"

Colegio de Defensa Nacional

Provincia La Habana:

Universidad Agraria de La Habana "Fructuoso Rodríguez" (UNAH)

Instituto Superior Pedagógico de La Habana "Rubén Martínez Villena"

${ }^{7}$ Las facultades de las demás provincias se adscriben a esta IES. 
Academia Naval "Granma"

Escuela Interarmas "General José Maceo"

Escuela Internacional de Educación Física y Deportes

Facultad de Cultura Física de La Habana

Universidad de las Ciencias Informáticas (UCI)

Provincia de Matanzas:

Universidad de Matanzas "Camilo Cienfuegos" (UMCC)

Instituto Superior Pedagógico "Juan Marinello"

Facultad de Ciencias Médicas de Matanzas

Facultad de Cultura Física de Matanzas

Provincia Las Villas:

Universidad Central de Las Villas "Marta Abreu" (UCLV)

Instituto Superior Pedagógico "Félix Varela"

Instituto Superior de Ciencias Médicas de Villa Clara

Facultad de Cultura Física de Villa Clara

Provincia Cienfuegos:

Universidad de Cienfuegos "Carlos Rafael Rodríguez"

Instituto Superior Pedagógico de Cienfuegos

Facultad de Ciencias Médicas de Cienfuegos

Facultad de Cultura Física de Cienfuegos

Provincia Sancti Spíritus:

Centro Universitario de Sancti Spíritus "José Martí" (CUSS)

Facultad de Ciencias Médicas de Sancti Spíritus

Instituto Superior Pedagógico "Capitán Silverio Blanco N:"

Facultad de Cultura Física de Sancti Spíritus

Provincia de Ciego de Ávila:

Universidad de Ciego de Ávila (UNICA)

Facultad de Ciencias Médicas de Ciego de Ávila

Instituto Superior Pedagógico "Manuel Ascunce Domenech"

Facultad de Cultura Física de Ciego de Ávila

Provincia de Camagüey:

Universidad de Camagüey (UC)

Instituto Superior de Ciencias Médicas de Camagüey

Instituto Superior Pedagógico "José Martí"

Facultad de Cultura Física de Camagüey 
Provincia Las Tunas:

Centro Universitario "Vladimir I. Lenin" (CULT)

Instituto Superior Pedagógico "Pepito Tey"

Facultad de Ciencias Médicas de Las Tunas

Facultad de Cultura Física de Las Tunas

Provincia de Holguín:

Universidad de Holguín "Oscar Lucero Moya" (UHOLM)

Instituto Superior Minero Metalúrgico de Moa (ISMMM)

Instituto Superior Pedagógico "José de la Luz y Caballero"

Facultad de Ciencias Médicas de Holguín

Facultad de Cultura Física de Holguín

Provincia Granma:

Universidad de Granma (UDG)

Instituto Superior Pedagógico "Blas Roca Calderío"

Facultad de Ciencias Médicas de Granma

Facultad de Cultura Física de Granma

Provincia de Santiago de Cuba:

Universidad de Oriente (UO)

Instituto Superior de Ciencias Médicas de Santiago de Cuba

Instituto Superior Pedagógico "Frank País García"

Facultad de Cultura Física de Santiago de Cuba

Provincia de Guantánamo:

Centro Universitario de Guantánamo (CUG)

Facultad de Ciencias Médicas de Guantánamo

Instituto Superior Pedagógico "Raúl Gómez García"

Facultad e Cultura Física de Guantánamo

Municipio Especial Isla de la Juventud:

Centro Universitario de la Isla de la Juventud "Jesús Montané Oropesa (CUIJ)

Filial de Ciencias Médicas de la Isla de la Juventud

Filial Pedagógica "Carlos Manuel de Céspedes"

Facultad de Cultura Física de la Isla de la Juventud 
ANEXO 2: Niveles de la Educación Superior cubana:

\begin{tabular}{|c|c|c|c|}
\hline \multirow{9}{*}{ POSTGRADO } & \multirow{5}{*}{ Formación Académica } & \multirow{2}{*}{$\begin{array}{l}\text { Grado Científico de } \\
\text { Doctor }\end{array}$} & Doctor en Ciencias \\
\hline & & & $\begin{array}{l}\text { Doctor en Ciencias en } \\
\text { Determinada Especialidad }\end{array}$ \\
\hline & & \multirow[t]{2}{*}{ Especialista } & Segundo Grado \\
\hline & & & Primer Grado \\
\hline & & \multicolumn{2}{|l|}{ Máster } \\
\hline & \multirow[t]{4}{*}{ Superación Profesional } & \multicolumn{2}{|c|}{$\begin{array}{l}\text { Posdoctorado (solo para profesionales con el } \\
\text { grado científico de Doctor) }\end{array}$} \\
\hline & & \multirow{2}{*}{\multicolumn{2}{|c|}{\begin{tabular}{|l|} 
Diplomado \\
Entrenamiento de Postgrado \\
\end{tabular}}} \\
\hline & & & \\
\hline & & \multicolumn{2}{|c|}{ Curso de Postgrado } \\
\hline PREGRADO & & \multicolumn{2}{|c|}{$\begin{array}{l}\text { Licenciado, Ingeniero, doctor en Medicina, doctor } \\
\text { en Medicina Veterinaria, Arquitecto }\end{array}$} \\
\hline
\end{tabular}

Fuente: Estudio sobre las certificaciones en la educación superior cubana (RAMOS, 2005).

\section{ANEXO 4: Matrícula Nacional de Pregrado por Institución y Género, curso 2005-2006.}

\begin{tabular}{|c|c|c|c|}
\hline \multirow[b]{2}{*}{ OACE: } & \multirow[b]{2}{*}{ Total } & \multicolumn{2}{|c|}{ Mujeres } \\
\hline & & Total & $\%$ \\
\hline MES & 57805 & 29146 & $50.42 \%$ \\
\hline MINED & 113244 & 80710 & $71.27 \%$ \\
\hline MINSAP & 89285 & 70428 & $78.88 \%$ \\
\hline OTROS & 21481 & 8155 & $37.96 \%$ \\
\hline SubTotal & 281815 & 188439 & $66.87 \%$ \\
\hline SUM & 165084 & 107305 & $65.00 \%$ \\
\hline SubTotal & 446899 & 295744 & $66.18 \%$ \\
\hline PREP & 577 & 289 & $50.00 \%$ \\
\hline EDIST & 40063 & 21634 & $54.00 \%$ \\
\hline Total & 487539 & 317666 & $65.16 \%$ \\
\hline
\end{tabular}

Fuente: Elaboración propia a partir de datos del Departamento de Estadísticas del MES, de la "Investigación sobre la Composición del Nuevo Ingreso a la Educación Superior" y consultas a IES.

ANEXO 5: Matrícula Nacional de Postgrado año 2005.

\begin{tabular}{|c|r|r|r|r|r|}
\hline Postgrado & \multicolumn{1}{|c|}{ TOTAL } & MES & MINED & MINSAP & \multicolumn{1}{c|}{ OTROS } \\
\hline Cursos & 385191 & 108629 & 106044 & 115289 & 55229 \\
\hline Diplomados & 76870 & 20706 & 33030 & 15578 & 7556 \\
\hline Entrenamientos & 20124 & 5360 & 2605 & 7127 & 5032 \\
\hline Maestría & 98795 & 10617 & 76455 & 10833 & 890 \\
\hline Especialidad & 14296 & 2564 & 91 & 11457 & 184 \\
\hline Doctorado & 4129 & 2145 & 965 & 452 & 567 \\
\hline TOTALES & 599405 & 150021 & 219190 & 160736 & 69458 \\
\hline
\end{tabular}

Fuente: Departamento de Estadísticas del MES (2005). 


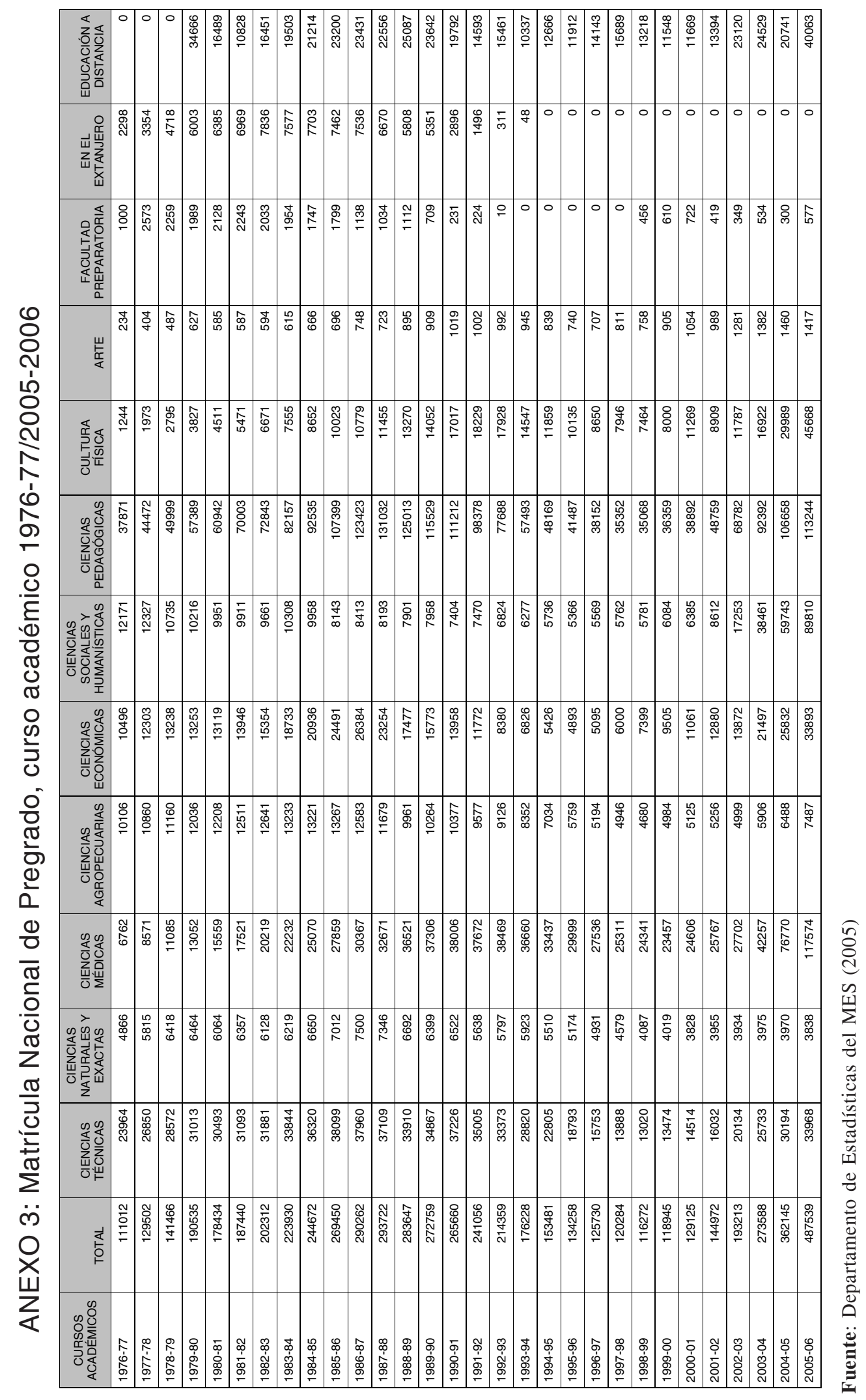




\section{REFERÊNCIAS}

CENTRO DE ESTUDIOS PARA EL PERFECCIONAMIENTO DE LA EDUCACIÓN SUPERIOR -CEPES. La educación superior cubana en la década del 90. La Habana: Editorial "Félix Varela", 2002.

Disponible em: www.iesalc.unesco.org.ve

GÓMEZ, Luis I. La nueva universidad y las estrategias para la universalización. La Habana: Palacio de las Convenciones, Febrero de 2006. Conferencia Magistral del Ministro de Educación, en el Congreso Universidad 2006. (Memorias Universidad 2006). ISBN 959-0282-08-3. INSTITUTO INTERNACIONAL PARA LA EDUCACIÓN SUPERIOR EN AMÉRICA LATINA Y EL CARIBE. - IESALC. Estudio sobre el financiamiento de la educación superior de Cuba. La Habana, diciembre de 2004. Disponible en: www.iesalc.unesco.org.ve INSTITUTO INTERNACIONAL PARA LA EDUCACIÓN SUPERIOR EN AMÉRICA LATINA Y EL CARIBE. - IESALC. Estudio sobre las certificaciones en la educación superior cubana. La Habana, mayo de 2005. Disponible en: www.iesalc.unesco.org.ve

LA UNIVERSIDAD Cubana: el modelo de formación. La Habana: Editorial "Félix Varela", 2006.

LÓPES SEGRERA, F. Cuba después del colapso de la Unión Soviética. México: UNAM / Centro de Investigaciones Interdisciplinarias en Ciencias y Humanidades, 1998.

OFICINA NACIONAL DE ESTADÍSTICA- ONE. VIII Censo Nacional de Población y Viviendas 2002. Republica Dominicana, Cuba, 2002. Disponible en: http://celade.cepal.org/cgibin/RpWebEngine.exe/ PortalAction?\&MODE=MAIN\&BASE=CPVDOM2002\&MAIN=WebServerMain.inl OFICINA NACIONAL DE ESTADÍSTICA - ONE. Panorama Económico y Social Cuba 2005 2006. La Habana, Cuba: ONE, 2006. Disponible en: http://www.one.cu/publicaciones/ultimas/ panorama2006.pdf

POLÍTICA de Ciencia y Tecnología y Política Económica y Social en Cuba. Intervención en el Congreso Universidad 2006. La Habana: Palacio de las Convenciones, feb. 2006. Memorias Universidad. ISBN 959-0282-08-3.

RAMOS SERPA, Gerardo (Coord.) Estudio sobre las certificaciones en la educación superior cubana. La Habana, Cuba: Centro de Estudio y Desarrollo Educacional, Universidad de Matanzas "Camilo Cienfuegos", 2005. 